\title{
The Topogical Model of the Chromatographic Retention Index of Nitrogen-Containing Polycyclic Aromatic Hydrocarbons
}

\author{
Yan Chen ${ }^{a}$ Changjun Feng ${ }^{b}$ Xiaotao Ding ${ }^{c}$ \\ School of Chemistry and Chemical Engineering, Xuzhou Institute of Technology, Xuzhou, Jiangsu, \\ 221111, China \\ achenyan681110@126.com, bfengcj@xzit.edu.cn ${ }^{\mathrm{c}} 1254533304 @ q q . c o m$
}

\begin{abstract}
Keywords: PANHs, topological index, chromatographic retention index, QSRR
Abstract: Based on the topological theory and MATLAB program, molecular connectivity index $\left(X_{i}\right)$, molecular shape index $\left(K_{i}\right)$, electrotopological state index $\left(E_{i}\right)$ and electro-negativity distance vector $\left(M_{i}\right)$ were calculated for 117 nitrogen-containing polycyclic aromatic hydrocarbons. A eight-element regression model of quantitative structure-retention relationship (QSRR) was constructed using leaps-and-bounds regression (LBR). The traditional correlation coefficient $(R)$, determination coefficient $\left(R^{2}\right)$ and the cross-validation correlation coefficient $\left(Q^{2}\right)$ were $0.992,0.985$ and 0.981 respectively. The robustness of the regression model was validated by Jackknife method, the correlation coefficient R was between 0.992 and 0.994 . Meanwhile, the model was further tested by external validation procedure, the calculated values of the compound in validation set were in good agreement with experimental data, the average relative error was 3.04\%. The regression results indicate that the model is highly reliable and has favorable predictive ability, and can better elucidate the change rule of retention indexes for nitrogen-containing polycyclic aromatic hydrocarbons.
\end{abstract}

\section{Introduction}

Polycyclic aromatic hydrocarbon (PAH) is a typical persistent organic pollutant, which is usually found in the petrochemical products, rubber, plastic, lubricating oil, antirust oil, incomplete combustion of organic compounds and other substances. The nitrogen-containing polycyclic aromatic hydrocarbons (PANHs) such as indol, quinoline, isoquinoline and their derivative exist widely in industrial waste came from petroleum industry, food industry, pesticide and pharmaceutical companies[1]. Because they are not easy to be degraded by organisms in the natural environment, They will do potential damage to the environment and human health[2]

In recent years, the quantitative structure-property/activity/retention relationship QSPR/QSAR /QSRR method was widely used in the prediction of physical and chemical properties, bioactivity and chromatographic properties of organic pollutants[3-6]. Among them, QSRR research has become a simple and effective method for the chromatographic research field, the results of the research work have also been fully recognized. On the basis of previous work[7, 8], molecular connectivity index $\left(X_{i}\right)$, molecular shape index $\left(K_{i}\right)$, electrotopological state index $\left(E_{i}\right)$ and electro-negativity distance vector $\left(M_{i}\right)$ were calculated using the MATLAB programs $[9,10]$, and through quantitative structureretention relationship analysis between these indexes and the chromatographic retention index of 117 PANHs, A topological model with good stability and predictive ability was established.

\section{Materials and Methods}

\section{Chromatographic retention index of PANHs}

117 PANHs were selected as research objects. The experimental values of their chromatographic retention index $(R I)$ were obtained from the literature[11]. The $R I$ values of 117 kinds of PANHs were shown in Table 1. 
Table 1 The chromatographic retention index $(R I)$ of 117 PANHs compounds

\begin{tabular}{|c|c|c|c|c|c|c|c|}
\hline \multirow{2}{*}{ No. } & \multirow{2}{*}{ PANHs } & \multicolumn{2}{|c|}{$R I$} & \multirow{2}{*}{ No. } & \multirow{2}{*}{ PANHs } & \multicolumn{2}{|c|}{$R I$} \\
\hline & & Exp. & Cal. & & & Exp. & Cal. \\
\hline 1 & 1-Aminoindan & 207.63 & 223.95 & 60 & 1,4-Dimethylcarbazole & 343.16 & 340.65 \\
\hline 2 & Quinolie & 210.26 & 212.61 & 61 & 2-Phenylindole & 346.18 & 344.26 \\
\hline 3 & Isoquinoline & 214.14 & 216.13 & *62 & 1,2-Dimethylcarbazole & 347.31 & 353.00 \\
\hline 4 & 1-Methylindole & 216.90 & 227.47 & 63 & 2-Azafluoranthene & 347.39 & 362.30 \\
\hline$* 5$ & Indole & 222.66 & 220.09 & 64 & 1-Azafluoranthene & 348.17 & 361.59 \\
\hline 6 & 7-Azaindole & 223.70 & 201.68 & 65 & 1,3-Dimethylcarbazole & 348.45 & 350.40 \\
\hline 7 & 2-Methylquinoline & 224.13 & 225.78 & *66 & 9-Cyanoanthracene & 350.46 & 359.94 \\
\hline 8 & 8-Methylquinoline & 225.18 & 228.13 & 67 & 7-Azafluoranthene & 350.50 & 362.31 \\
\hline 9 & 1-Methylisoquinoline & 229.21 & 228.38 & 68 & 9-Cyanophenanthrene & 351.84 & 356.76 \\
\hline *10 & 7-Methylquinoline & 231.37 & 232.68 & 69 & 2-Nitrofluorene & 353.06 & 354.35 \\
\hline 11 & 5-Aminoindan & 232.12 & 243.62 & 70 & 4-Aminophenanthrene & 353.97 & 345.14 \\
\hline 12 & 3-Methylquinoline & 232.47 & 230.37 & 71 & 9-Nitroanthracene & 357.42 & 364.13 \\
\hline 13 & 7-Methylindole & 235.49 & 240.97 & *72 & 1-Azapyrene & 357.73 & 369.83 \\
\hline$* 14$ & 4-Methylquinoline & 235.77 & 229.69 & 73 & 4-Azapyrene & 357.94 & 372.19 \\
\hline 15 & 3-Methylindole & 239.20 & 239.72 & 74 & 2-Azapyrene & 362.43 & 367.48 \\
\hline 16 & 2-Methylindole & 240.10 & 234.29 & 75 & 1-Aminophenanthrene & 362.62 & 347.13 \\
\hline 17 & 2,7-Dimethylquinoline & 244.04 & 245.37 & 76 & 1-Aminoanthracene & 362.83 & 355.81 \\
\hline 18 & 2,6-Dimethylquinoline & 244.19 & 245.95 & 77 & 9-Aminophenanthrene & 362.83 & 347.65 \\
\hline 19 & 1,2-Dimethylindol & 244.42 & 249.90 & 78 & 9-Aminoanthracene & 363.91 & 364.13 \\
\hline *20 & 2, 2-Bipyridyl & 247.15 & 221.46 & 79 & Benzo[def]carbazole & 363.92 & 368.72 \\
\hline 21 & 2,4-Dimethylquinoline & 247.96 & 245.00 & $* 80$ & 3-Aminophenanthrene & 365.60 & 352.94 \\
\hline *22 & 4-Azabiphenyl & 252.35 & 246.99 & 81 & 2-Aminophenanthrene & 365.80 & 353.30 \\
\hline 23 & 2,5-Dimethylindole & 256.65 & 256.61 & 82 & 2-Aminoanthracene & 367.45 & 361.41 \\
\hline 24 & 1-Cyanonapythalene & 256.75 & 264.03 & 83 & 3,5-Diphenylpyridine & 372.84 & 372.59 \\
\hline 25 & 2,3-Dimethylindole & 257.32 & 259.81 & $* 84$ & 9-Phenylcarbazole & 381.51 & 400.80 \\
\hline$* 26$ & 2-Cyanonapythalene & 260.88 & 266.37 & 85 & Benz[c]acridine & 392.60 & 403.99 \\
\hline 27 & 5-Nitroindan & 261.55 & 266.24 & 86 & Benz[a]acridine & 398.65 & 405.08 \\
\hline 28 & 1-Aminopaphthalene & 262.98 & 250.37 & 87 & 1-Azabenz[a]anthracene & 400.00 & 411.20 \\
\hline 29 & 2-Aminoaphthalene & 265.53 & 255.79 & $* 88$ & 4-Azachrysene & 401.16 & 403.02 \\
\hline$* 30$ & 2,3,5-Trimethylindole & 273.61 & 283.18 & 89 & Benzo[a]carbazole & 402.22 & 405.71 \\
\hline 31 & 2-Aminobiphenyl & 237.63 & 279.16 & 90 & 1-Azachrysene & 407.18 & 404.45 \\
\hline 32 & 1-Nitronaphthalene & 274.95 & 271.19 & 91 & Benzo[b]carbazole & 409.63 & 414.20 \\
\hline 33 & 4-Azafluorene & 279.85 & 283.14 & 92 & 3-Aminofluoranthene & 409.97 & 403.33 \\
\hline *34 & 2-Nitronaphthalene & 280.63 & 278.97 & 93 & 2-Azachrysene & 411.49 & 408.69 \\
\hline 35 & 3-Methyl-2-aminonaphthalene & 283.73 & 281.91 & 94 & Benzo[c]carbazole & 411.89 & 401.26 \\
\hline 36 & 2-Nitrobiphenyl & 290.25 & 300.46 & 95 & 4-Aminopyrene & 412.31 & 410.78 \\
\hline *37 & Phenazine & 294.37 & 282.80 & *96 & 2-Aminopyrene & 413.83 & 417.92 \\
\hline 38 & 4-Aminobiphenyl & 298.05 & 283.74 & 97 & 1-Aminopyrene & 415.39 & 410.65 \\
\hline 39 & Benzo[h]quinoline & 301.94 & 306.68 & 98 & 1-Nitropyrene & 421.48 & 429.66 \\
\hline 40 & Acridine & 304.04 & 309.11 & 99 & 2,2-Biquinoline & 422.56 & 411.15 \\
\hline$* 41$ & Acridan(9,10-dihydroacridine) & 304.11 & 318.07 & 100 & 7,9-Dimethylbenz[c]acridine & 438.32 & 435.75 \\
\hline 42 & Benzo[f]quinoline & 307.94 & 307.76 & 101 & 5,7-Dimethylbenz[a]acridine & 438.38 & 434.78 \\
\hline 43 & Phenanthridine & 307.94 & 305.12 & *102 & 7,10-Dimethylbenz[a]acridine & 439.46 & 433.30 \\
\hline 44 & 3-Nitrobiphenyl & 310.09 & 307.61 & 103 & 2-Aminobenzo[c]phenanthrene & 450.10 & 448.66 \\
\hline 45 & Carbazole & 311.71 & 305.56 & 104 & 4-Aminobenzo[c]phenanthrene & 451.51 & 442.69 \\
\hline *46 & 4-Nitrobiphenyl & 314.59 & 308.52 & 105 & 10-Azabenzo[a]pyrene & 455.40 & 466.33 \\
\hline 47 & 3-Methylbenzo[f]quinoline & 320.26 & 320.37 & 106 & 6-Aminochrysene & 463.19 & 444.95 \\
\hline 48 & 2-Methylbenzo[f]quinoline & 320.50 & 324.76 & 107 & 9,10,12-Trimethylbenz[a]acridine & 466.79 & 463.30 \\
\hline$* 49$ & 2-Methylacridine & 324.34 & 329.56 & 108 & Dibenz[a,c]phenazine & 474.08 & 461.44 \\
\hline 50 & 1-Methylcarbazole & 324.45 & 325.68 & *109 & 5-Aminochrysene & 487.88 & 442.58 \\
\hline 51 & 4-Aminofluorene & 325.11 & 320.66 & 110 & Dibenz[a,h]acridine & 488.55 & 500.40 \\
\hline 52 & 1-Aminofluorene & 327.21 & 324.90 & 111 & Dibenzo[a,i]carbazole & 490.57 & 506.27 \\
\hline 53 & 3-Methylcarbazole & 328.81 & 327.46 & 112 & Dibenz[a,j]acridine & 490.66 & 501.42 \\
\hline *54 & 3-Aminofluorene & 329.08 & 330.92 & 113 & 6-Nitrobenzo[a]pyrene & 501.71 & 514.46 \\
\hline 55 & 2-Methylcarbazole & 329.61 & 328.33 & 114 & Dibenzo[a,g]carbazole & 502.30 & 501.80 \\
\hline 56 & 9-Methylacridine & 331.15 & 321.50 & *115 & Dibenzo[c,g]carbazole & 502.92 & 496.75 \\
\hline 57 & 4-Methylcarbazole & 331.88 & 320.93 & 116 & 7-Aminobenzo[a]pyrene & 511.98 & 509.09 \\
\hline *58 & 2-Aminofluorene & 331.91 & 332.11 & 117 & 6-Aminobenzo[a]pyrene & 515.66 & 500.05 \\
\hline 59 & 6-Phenylquinoline & 340.84 & 344.28 & & & & \\
\hline
\end{tabular}




\section{Construction method of topological index}

Topological index method is one of the most convenient methods for QSPR/QSRR/QSAR research. At present, the more commonly used topological index are molecular connectivity index, molecular shape index, electrotopological state index and molecular electronegativity distance vector, which are constructed based on the method of graph theory.

Using Chem3D Ultra 9.0, molecule structures of 117 PANHs were built, and saved as .mol file, then called the above files, in the MATLAB environment, 10 molecular connectivity indexes, 4 molecular shape indexes, 46 electrotopological state indexes and 91 molecular electronegativity distance vector were calculated by MATLAB programs. We got 151 topological indexes as molecular descriptors.

\section{Screening of molecular descriptors}

According to the principle of statistics, the number of variables is less than $5 \%$ of the independent variable, and its contribution to the dependent variable can be neglected. Therefore, the independent variables which number was less than $6(117 \times 5 \%)$ were removed, and the remaining 26 topological indexes were used to characterize the molecular structure of 117 PANHs.

\section{Multiple linear regression method}

Using the remaining 26 topological indexes of 117 PANHs as independent variables, and the chromatographic retention index as dependent variables, We had chosen the best variables of relativity with Chromatographic properties by MINITAB 14 software, and then built up the quantitative structure- retention relationship (QSRR) mathematical model between these topological index and RI. Meanwhile, Kubinyi (function FIT, Kubinyi) [12-13] was introduced to judge the stability and prediction ability of the model, that the calculation formula is:

$$
\mathrm{FIT}=\frac{R^{2}(y-b-1)}{\left(y+b^{2}\right)\left(1-R^{2}\right)}
$$

In the formula, $y$ is the sample size of the compounds, $b$ is the number of variables. The bigger is FIT, the more stable is the model, and the better is the ability of prediction.

\section{Results and discussion}

\section{QSRR equation of the $R I$}

By employing MINITAB 14.0 program, leaps-and-bounds regression method was carried out, with the results between $R I$ and topological indexes of 117 PANHs presented in Table 2.

Table 2 Results of the topological index and RI with the leaps-and-bounds regression

\begin{tabular}{cccccccc}
\hline No. & $R$ & $R^{2}$ & $R_{a d j}{ }^{2}$ & $S$ & $F$ & $F I T$ & Variables \\
\hline 1 & 0.955 & 0.913 & 0.912 & 24.298 & 1203.295 & 9.173 & $X_{1}$ \\
2 & 0.983 & 0.966 & 0.965 & 15.267 & 1613.367 & 26.768 & $X_{1}, M_{15}$ \\
3 & 0.986 & 0.971 & 0.971 & 14.018 & 1283.190 & 30.028 & $X_{1}, M_{15}, M_{6}$ \\
4 & 0.988 & 0.976 & 0.975 & 12.887 & 1144.112 & 34.246 & $X_{1}, M_{15}, M_{6}, M_{18}$ \\
5 & 0.989 & 0.978 & 0.977 & 12.337 & 1000.929 & 34.750 & $X_{1}, M_{15}, M_{6}, M_{18}, K_{1}$ \\
6 & 0.990 & 0.980 & 0.979 & 11.820 & 910.557 & 35.229 & $X_{1}, M_{15}, M_{6}, M_{18}, K_{1}, E_{19}$ \\
7 & 0.991 & 0.982 & 0.980 & 11.464 & 830.847 & 35.932 & $X_{1}, M_{15}, M_{6}, M_{18}, K_{1}, E_{19}, M_{2}$ \\
8 & 0.992 & 0.984 & 0.983 & 10.601 & 852.633 & 36.696 & $X_{1}, M_{15}, M_{6}, M_{18}, K_{1}, E_{19}, M_{2}, M_{14}$ \\
9 & 0.992 & 0.985 & 0.984 & 10.462 & 778.573 & 35.486 & $X_{1}, M_{15}, M_{6}, M_{18}, K_{1}, E_{19}, M_{2}, M_{14}, X_{12}$ \\
\hline
\end{tabular}

where $R$ is the traditional correlation coefficient, $R^{2}$ is the determination coefficient, $R_{\mathrm{adj}}{ }^{2}$ is the square of adjusted correlation coefficient, $S$ is the standard deviation of the regression and $F$ is the Fisher ratio. From Table 2, The FIT value gradually increased, and the turning point was 36.696, which showed that the eight element model had the best stability and prediction ability. Corresponding multiple regression equations were shown as follows:

$R I=27.535+48.059 X_{1}-24.068 K_{1}+4.343 E_{19}-4.759 M_{2}-7.641 M_{6}-0.908 M_{14}+1.703 M_{15}-$ 


$$
n=117, R=0.992, R^{2}=0.984, S=10.601, F=778.573
$$

The calculated values of $R I$ given by the formula (2) were listed in Table 1 , which were in agreement with the corresponding experimental values. The average relative error was $2.22 \%$.

\section{Stability test of the model}

\section{LOO cross validation of the model}

By LOO cross validation correlation coefficient $\left(Q^{2}\right)$ was 0.982 and slightly smaller than 0.985 ; cross validation standard deviation was 10.682, slightly larger than 10.601 This shows that the stability and prediction ability of the model are ideal.

\section{Jackknifed test of the model}

To test whether there was any "abnormal value” in model (2), we carried through the stability test based on Jackknifed method[14]. Concerning the researched was big samples (the capability was more than 30), we applied the elimination way group by group. Namely, every time we eliminated the compounds whose serial number contains $1,2,3 \ldots 0$ on unit order in the sample, and then established the model with the rest compounds' RI. The average value of these 10 correlation coefficients was 0.992, which was consistent with the model (2). and R's fluctuating extension was very small. Fig 1 was control graph of 10 correlation coefficients. From Figure 1, all values were in the control area (between 0.9905 and 0.9940.)it suggest that the eight element model has good robustness.

\section{External validation of the model}

In order to further verify the robustness of the model, we texted the model by an external validation. The 117 samples were divided into the training set and the test set. The training set was used to set up the model, and the test set was used to be predicted. We selected 93 compounds randomly as training set samples, the remaining 24 compounds (with * mark in Table 1) as the external test set sample. The predicted values of the retention index of the test set was close to the experiment values.

The model of the training set was used to estimate the chromatographic retention index, the average relative error was 3.04\%. Fig 2 was the plot of predicted against experimental values of chromato- graphic retention indexes

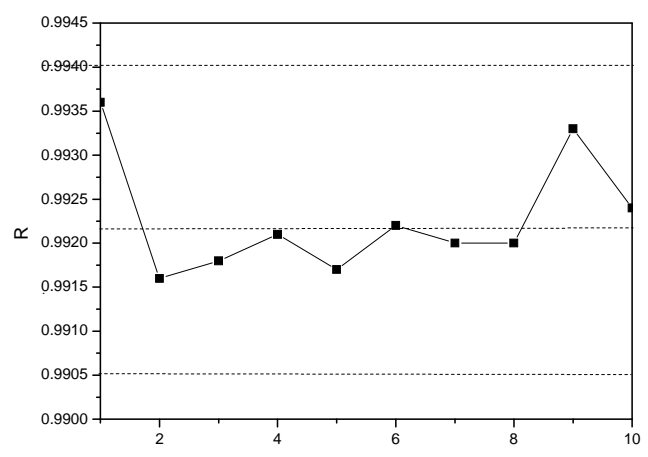

Fig.1 The control chart of Jackknifed correlation coefficient

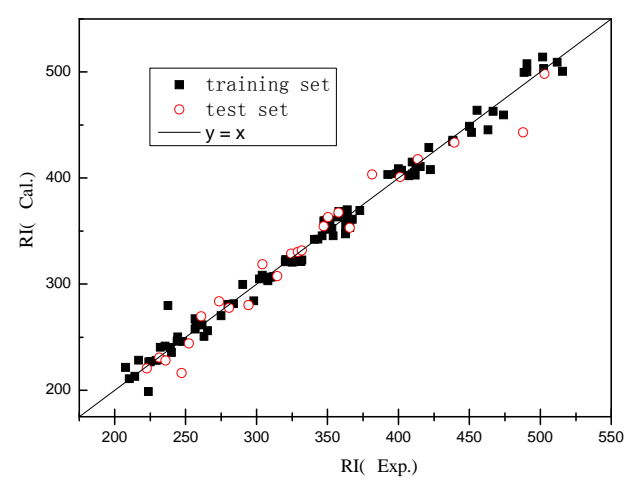

Fig.2 Plot of calculated against experimental values of retention indexes

\section{Conclusion}

There are a lot of factors affecting gas chromatography retention index, but the molecular interaction including dispersion force, induction force, orientation force and hydrogen bond between the components and the stationary phase is the main factor. Nitrogen containing polycyclic aromatic hydrocarbons are weakly polar molecules, so the molecular interaction between molecules is mainly based on the dispersion forces. The $X_{1}$ and $K_{1}$ in the model reflect the size and shape of the molecules, so the value can be used to characterize the dispersion force. $\mathrm{E}_{19}$ corresponding to the structure of aNa ( $\mathrm{a}$ is the conjugated bonds in the aromatic ring), that is, the nitrogen atoms in the ring, the polar 
groups, can be used to characterize the induction force and orientation force, At the same time, the electronegativity of the nitrogen atom is relatively large, so when the stationary phase has active hydrogen atom, it can form hydrogen bonds, which also can be used to characterize the hydrogen bonds. $M_{2}, M_{6}, M_{14}, M_{15}, M_{18}$ are the interaction between the first class atom $\left(\mathrm{CH}_{3^{-}}\right)$and second class atom $\left(-\mathrm{CH}_{2}-\right)$, the first class atom and the sixth class atom(-N-), the second class atom and the second class atom, the second class atom and the fourth class atom $(>\mathrm{C}<)$, the second class atom and the sixth class atom, respectively. $M_{14}, M_{15}$ and $M_{2}$, the interaction between polar groups, can be used to characterize the dispersion force, and $M_{18}, M_{6}$, the interaction between polar groups and non polar groups, can be used to characterize the induction force and orientation force. So the molecular connectivity index, molecular shape index, electrotopological state index and molecular electronegativity distance vector reveal the factors affecting gas chromatography retention index of PANHs. The cut error ratio (i.e., $\mathrm{R}^{2}$ ) of the model (2) is $98.4 \%$, and only $1.6 \%$ of the other factors that affect the RI are not disclosed.

In summary, the model has good correlation, robustness and disclose the essential factors that affect the gas chromatographic retention index of the compounds. It is reasonable that four kinds of topological indices are used to characterize the molecular structures of the PANHs.

\section{Acknowledgements}

This work was financially supported by the National Natural Science Foundation of China (No. 21272095)

\section{References}

[1] Z D Xu, R H Shi and M Geng. J China Agric. Univ. Vol. 13 (2008), 57-62 (in Chinese) .

[2] M Jiang, Y M Li, G W Gu. Acta Sci. Circumst. Vol. 25(2005), 1253-1258 (in Chinese).

[3] C J FENG, W H Yang. Chinese J. Struct. Chem., Vol. 33(2014):830-834 (in Chinese).

[4] C J FENG, W H Yang, L L Mu. Chinese J. Struct. Chem., Vol. 27(2008):575-587 (in Chinese).

[5] C Wang, W Wang, L Q Pan, et al. Chem. Vol. 76 (2013): 929- 934 (in Chinese).

[6] Y Chen, W Yue, B Wang. J. Wuhan Univ.( Nat. Sci.Ed.), Vol. 60(2014): 52-56 (in Chinese).

[7] Y Chen. Bull Sci Technol, Vol. 29 (2013):16-19 (in Chinese).

[8] Y Chen. Food Sci.Vol. 32(2011):274-277 (in Chinese).

[9] Q. N. Hu, Y. Z. Liang, Y. L.Wang. Comp. App. Chem. Vol.20 (2003):386-390 (in Chinese).

[10] T. Zhang, Y.Z. Liang, C.X. Zhao. Chin. J. Anal. Chem. Vol. 34(2006):1607-1610 (in Chinese).

[11] C Wang, W Wei, L Q Pan, et, al. Chem. Vol.76(2013): 929-934 (in Chinese).

[12] L. H. Hall, L. B.Kier. J. Chem. Inform. Mod. Vol. 35(1995):1039-1045.

[13] L. S.Urra, M. P.Gonzalez, M.Teijeira. Bio. Med. Chem., Vol. 15(2007): 3565-3571.

[14] W S Dietrich, N D Dreyer, C Hansch. J Med Chem, Vol. 23(1980): 1201- 1205 